\title{
The Relationship between Lipoprotein a and Glycosylated Hemoglobin and the Degree of Coronary Artery Stenosis in Non-Diabetic Coronary Heart Disease Patients
}

\author{
Wangzhou ${ }^{1 \#}$, Chen Xuejinn", Qi Chunmei ${ }^{2 *}$, Wang Lele², Sun Hua Mei $^{2}$, Wu Jing Yu ${ }^{2}$ Fan Xiao \\ Dan $^{2}$, Jin Jing Jing ${ }^{2}$ and Dai Ya Nan ${ }^{2}$ \\ ${ }^{1}$ Xuzhou medical university, China \\ ${ }^{2}$ The Second Affiliated Hospital of Xuzhou medical university, China \\ ${ }^{\#}$ Contributed equally to this work. \\ *Corresponding author: Qi Chunmei, The Second Affiliated Hospital of Xuzhou medical university, China
}

\section{ARTICLE INFO}

Received: 慧 September 16, 2019

Published: 㹃 September 26, 2019

Citation: Wangzhou, Chen Xuejin, Qi Chunmei, Wang Lele, Sun Hua Mei. The Relationship between Lipoprotein a and Glycosylated Hemoglobin and the Degree of Coronary Artery Stenosis in Non-Diabetic Coronary Heart Disease Patients. Biomed J Sci \& Tech Res 21(4)2019. BJSTR. MS.ID.003624.

Abbreviations: CHD: Coronary Heart Disease; ACS: Acute Coronary Syndrome; SCD: Stable Coronary Heart Disease; HbA1c: Hemoglobin A1c; Lp(a): Lipoprotein a; Fbg: Fibrinogen; Hcy: Homocysteine; CRP: C-Reactive Protein; AS: Atherosclerosis; TG: Triglyceride; FBG: Fasting Blood Glucose

\section{ABSTRACT}

Objectives: To explore the relationship between lipoprotein a (Lp (a)), glycosylated hemoglobin (HbA1c) and coronary stenosis in patients with non - diabetic coronary heart diseases. Methods: A total of 302 patients with non-diabetic coronary heart disease were randomly selected from January 2018 to February 2019 in the department of cardiology of our hospital according to the random number table method. Biochemical, electrocardiogram and other relevant test results as well as general characteristics such as address, sex and age of the selected patients were recorded .Serum levels of Lp (a) and $\mathrm{HbA1c}$ were determinate and analyzed by One-way Anova to explore the correlation between them and the degree of coronary artery diseases.

Results: One-way Anova indicated that there were significant differences of coronary Gensini score among different serum level of Lp (a) groups. However, there was no statistically significant difference in the coronary Gensini score among the three groups of patients with different serum HbA1c levels $(\mathrm{P}=0.500)$.Spearman correlation analysis showed that coronary Gensini score was positively correlated with Lp (a) ( $\mathrm{r}=0.284, \mathrm{P}<$ $0.001)$. There was no significant relationship between coronary Gensini score and HbA1c $(\mathrm{P}=0.500)$. There was no correlation between HbA1c and Lp (a) $(\mathrm{r}=0.103, \mathrm{P}=0.075)$.

Conclusion: HbA1c level has not obviously the correlation with the degree of coronary artery stenosis, but Lp(a) level is positively correlated with the degree of coronary artery stenosis and has a predictive value for the degree of coronary artery disease in non - diabetic CHD individuals.

Keywords: Non-Diabetes; Coronary Heart Disease (CHD); Lipoprotein(a) [Lp (a)]; Hemoglobin A1c(HbA1c); Gensini Score

\section{Introduction}

Nowadays, with the rapid development of society and economy, people's living standard is constantly improved, and unhealthy lifestyle is also prevalent. As a result, the incidence of coronary heart disease increases year by year and tends to be younger. It has become one of the most important diseases endangering human life and health. Hyperglycemia, hyperlipidemia, hypertension and other factors are closely related to coronary heart disease. It was found that lipoprotein a $(\operatorname{Lp}(\mathrm{a}))$ and glycosylated hemoglobin (HbA1c) are one of the major risk factors for coronary heart disease. AS a gold indicator of blood glucose control level, HbA1c can reflect the blood glucose level of nearly 3 months, which is generally believed to be closely related to the occurrence and development 
of atherosclerosis (AS) [1-3]. In recent years, domestic and foreign research data show that $\mathrm{HbA1c}$ is a risk factor for coronary heart disease in patients with type 2 diabetes [4-5]. In addition, related studies have shown that Lp (a) level is also closely related to the occurrence and development of atherosclerosis [6]. In this study, 302 patients with non-diabetic coronary heart disease were selected to detect their Lp(a) level and HbA1c. Gensini score was used as the criterion to evaluate the degree of coronary stenosis. The purpose of this study was to explore whether the level of $\mathrm{Lp}(\mathrm{a})$ and HbA1c in non-diabetic coronary heart disease patients affected the development of coronary heart disease and the relationship with the degree of coronary stenosis.

\section{Patient Selection and Diagnosis}

A total of 302 patients with non-diabetic coronary heart disease were admitted to the department of cardiology of our hospital from January 2018 to February 2019. According to the American Diabetes Association (ADA) diagnostic criteria for DMs in 2016:

a) The level of fasting blood glucose is higher than 7.0 $\mathrm{mmol} / \mathrm{L}$;

b) The level of blood glucose is higher than $11.1 \mathrm{mmol} / \mathrm{L}$ two hours after OGTT test;

c) $\mathrm{HbA1c}$ is greater than or equal to $6.5 \%$;

d) The typical symptoms of hyperglycemia, the risk of hyperglycemia and the patients with blood glucose greater than or equal to $11.1 \mathrm{mmol} / \mathrm{L}$ at random time;

e) The patients with glucose before OGTT test. Urinary history is currently under dietary control and drug treatment.

Anyone who meets any of the above criteria can diagnose DM is excluded from the study. Exclusion criteria:

a) Patients diagnosed with recurrent myocardial infarction.

b) Patients who had undergone coronary stent implantation.

c) Patients diagnosed with malignant tumors.

d) There are liver and kidney damage, such as elevated ALT elevations, elevated CREA, BUN, Cyc, rise).

e) Patients with other diseases affecting lipid metabolism, such as thyroid disease, nephrotic syndrome, etc.

f) Patients who have taken lipid-lowering drugs (Statins, etc.) for lipid-lowering treatment.

\section{Grouping}

The patients were grouped according to two methods:

a) according to the level of $\mathrm{Lp}(\mathrm{a})$, they were divided into three groups: group A, Lp (a) < 200mg/L ( $=145)$, group B, $200<\mathrm{Lp}(\mathrm{a})<300 \mathrm{mg} / \mathrm{L}(\mathrm{n}=73)$, and group C, Lp (a) $>300 \mathrm{mg} / \mathrm{L}$ $(\mathrm{n}=84)$. b) According to the level of HbA1c, they were divided into three groups from low to high, respectively: group I, HbA1c < $5.6 \mathrm{mmol} / \mathrm{L}(\mathrm{n}=130)$, group II, $5.7<\mathrm{HbA1c}<5.9 \mathrm{mmol} / \mathrm{L}(\mathrm{n}=$ 99), group III, $6.0<\mathrm{HbA1c}<6.5 \mathrm{mmol} / \mathrm{L}(\mathrm{n}=73)$.

\section{Methods}

\section{General Data}

Such as sex, age, history of hypertension, smoking history, etc.

\section{Blood Sample Collection}

The subjects were all fasting for more than 8 hours. The peripheral venous blood was collected $5 \mathrm{ml}$ the next morning to detect triglyceride (TG), cholesterol (TC), high density lipoprotein cholesterol (HDL-C), low density lipoprotein cholesterol (LDL-C), Lp (a), fasting blood glucose(FBG), HbA1c and other indicators.

\section{Coronary Angiography}

Coronary angiography shall be performed by senior physicians or above; The radial artery puncture approach was performed under local anesthesia, and the optimal exposure position was selected for examination under the guidance of digital angiography machine (Axiom Artis FA, Siemens, Germany). All patients underwent angiography of right coronary artery, left main artery, left circumflex artery and left anterior descending artery.

\section{Gensini Score}

According to the results of angiography, all coronary arteries and their branches were quantitatively assessed by Gensini score criteria:

a) According to the degree of stenosis, $1 \%-25 \%$ was 1 point, $26 \%-50 \%$ was 2 points, $51 \%-75 \%$ was 4 points, $76 \%-90 \%$ was 8 points, $91 \%-99 \%$ was 16 points and $100 \%$ was 32 points.

b) Coefficient of lesion location: left main coronary artery * 5; proximal anterior descending branch $* 2.5$, middle $* 1.5$; apical branch and first diagonal branch $* 1$, second diagonal branch $* 0.5$; proximal circumflex branch $* 2.5$, blunt marginal branch, distal circumflex branch and posterior descending branch * 1 , posterior branch * 0.5 ; proximal, middle and distal right coronary artery * 1 and posterior descending branch * 1 .

c) The lesion score $=$ the score of each coronary artery * the coefficient of the lesion site. If the patient has multiple vascular lesions, the total score of each lesion is the total score of the severity of coronary lesions.

\section{Statistical Analysis}

SPSS 21.0 software was used for statistical analysis. For measurement data, homogeneity test of variance and normality test were carried out. Data with homogeneity of variance and normal distribution among groups were presented as mean $\pm \mathrm{sd}$. The differences of data among groups were compared by oneway Anova. For data that do not conform to normal distribution, 
the median (quartile spacing) is presented, and kruskal-wallis test is adopted. For counting data, chi-square test is used. Spearman correlation analysis was used to analyze the relationship between

Results

\section{Comparison of Clinical Data between Groups with Different Lp(a) Levels}

Table 1: Comparison of clinical data of different serum lipoprotein a concentration group.

\begin{tabular}{|c|c|c|c|c|}
\hline & Group A $(n=145)$ & Group B $(n=73)$ & Group C $(n=84)$ & $\mathbf{P}$ \\
\hline Gender & $100(69.0 \%)$ & 55 (75.3\%) & $61(72.6 \%)$ & 0.595 \\
\hline Age & $62.29 \pm 9.98$ & $60.48 \pm 11.82$ & $62.43 \pm 11.81$ & 0.450 \\
\hline Hypertension & $85(58.6 \%)$ & $44(60.2 \%)$ & $44(52.4 \%)$ & 0.549 \\
\hline Smoking & $83(57.2 \%)$ & $41(56.2 \%)$ & $50(59.5 \%)$ & 0.506 \\
\hline $\mathrm{HbA1c}$ & $5.67 \pm 0.34$ & $5.67 \pm 0.38$ & $5.77 \pm 0.36$ & 0.086 \\
\hline TG & $1.43 \pm 0.97$ & $1.42 \pm 0.88$ & $1.50 \pm 0.71$ & 0.799 \\
\hline $\mathrm{TC}$ & $4.74 \pm 1.20$ & $4.75 \pm 0.91$ & $4.97 \pm 1.11$ & 0.274 \\
\hline LDL-C & $2.81 \pm 1.00$ & $2.88 \pm 0.87$ & $3.06 \pm 1.06$ & 0.178 \\
\hline HDL-C & $1.25 \pm 0.31$ & $1.20 \pm 0.26$ & $1.25 \pm 0.52$ & 0.675 \\
\hline FBG & $5.28 \pm 0.44$ & $5.32 \pm 0.43$ & $5.32 \pm 0.60$ & 0.772 \\
\hline Gensini Score & $47.07 \pm 35.15$ & $63.25 \pm 30.96$ & $73.31 \pm 33.61$ & $<0.001$ \\
\hline
\end{tabular}

There was no statistically significant difference in the clinical data of the three groups, such as age, smoking history, hypertension history, blood lipid and fasting blood glucose ( $>$ > 0.05). The mean Gensini score in serum Lp(a) group was higher than that in serum Lp(a) group $(\mathrm{P}<0.001)$ (Table 1$)$.

\section{Comparison of Gensini Scores between Groups with Different Lp(a) Levels}

One-way Anova was performed on Gensini scores of the three groups with different serum $\operatorname{Lp}(\mathrm{a})$ levels, and the results showed that serum $L p(a)$ levels were increased, while Gensini scores were increased, and the differences were statistically significant $(\mathrm{P}<$
Lp(a) and HbA1c coronary artery stenosis. The difference was statistically significant with $\mathrm{P}<0.05$.
0.001). Spearman correlation analysis was adopted to gradually adjust the confound factors influencing Gensini score, and Gensini score was still correlated with Lp(a) level ( $\mathrm{r}=0.286, \mathrm{P}<0.001)$.

\section{Comparison of Clinical Data between Groups with Different HbA1c Levels}

The average age of the high-level group was the largest, followed by the middle-level group and the low-level group $(\mathrm{P}=0.009)$. The FBG of the high-level group was higher than that of the middle-level group and higher than that of the low-level group ( $\mathrm{P}=0.01)$.TC and LDL-C in HbA1c medium-level group were higher than those in high-level group and low-level group $(\mathrm{P}=0.007, \mathrm{P}=0.023)$ (Table 2).

Table 2: Comparison of clinical data in groups with different serum hba1c concentrations.

\begin{tabular}{|c|c|c|c|c|}
\hline & Group I $(n=130)$ & Group II (n=99) & Group III $(n=73)$ & $\mathbf{P}$ \\
\hline Gender & $94(72 . \%)$ & $76(79.8 \%)$ & $46(63 \%)$ & 0.137 \\
\hline Age & $59.89 \pm 11.80$ & $62.42 \pm 10.00$ & $64.73 \pm 10.08$ & 0.009 \\
\hline Hypertension & $79(60.8 \%)$ & $52(52.5 \%)$ & $42(57.5 \%)$ & 0.458 \\
\hline Smoking & $76(58.5 \%)$ & $61(61.6 \%)$ & $37(50.7 \%)$ & 0.346 \\
\hline Lp(a) & $203.50(139.00-288.25)$ & $198.00(141.00-330.33)$ & $215.00(153.00330 .00)$ & 0.272 \\
\hline TG & $1.17(0.85-1.67)$ & $1.30(1.00-1.78)$ & $1.34(0.98-1.76)$ & 0.181 \\
\hline $\mathrm{TC}$ & $4.59 \pm 0.96$ & $5.04 \pm 1.27$ & $4.87 \pm 1.05$ & 0.007 \\
\hline LDL-C & $2.74 \pm 0.91$ & $3.10 \pm 1.13$ & $2.90 \pm 0.88$ & 0.023 \\
\hline HDL-C & $1.24 \pm 0.29$ & $1.26 \pm 0.50$ & $1.20 \pm 0.27$ & 0.583 \\
\hline FBG & $5.21 \pm 0.46$ & $5.32 \pm 0.58$ & $5.42 \pm 0.35$ & 0.010 \\
\hline Gensini Score & $57.16 \pm 39.42$ & $57.96 \pm 30.58$ & $60.71 \pm 34.74$ & 0.500 \\
\hline
\end{tabular}

Comparison of Gensini Scores in Different HbA1c Levels

he groups with high HbA1c had the highest Gensini score 


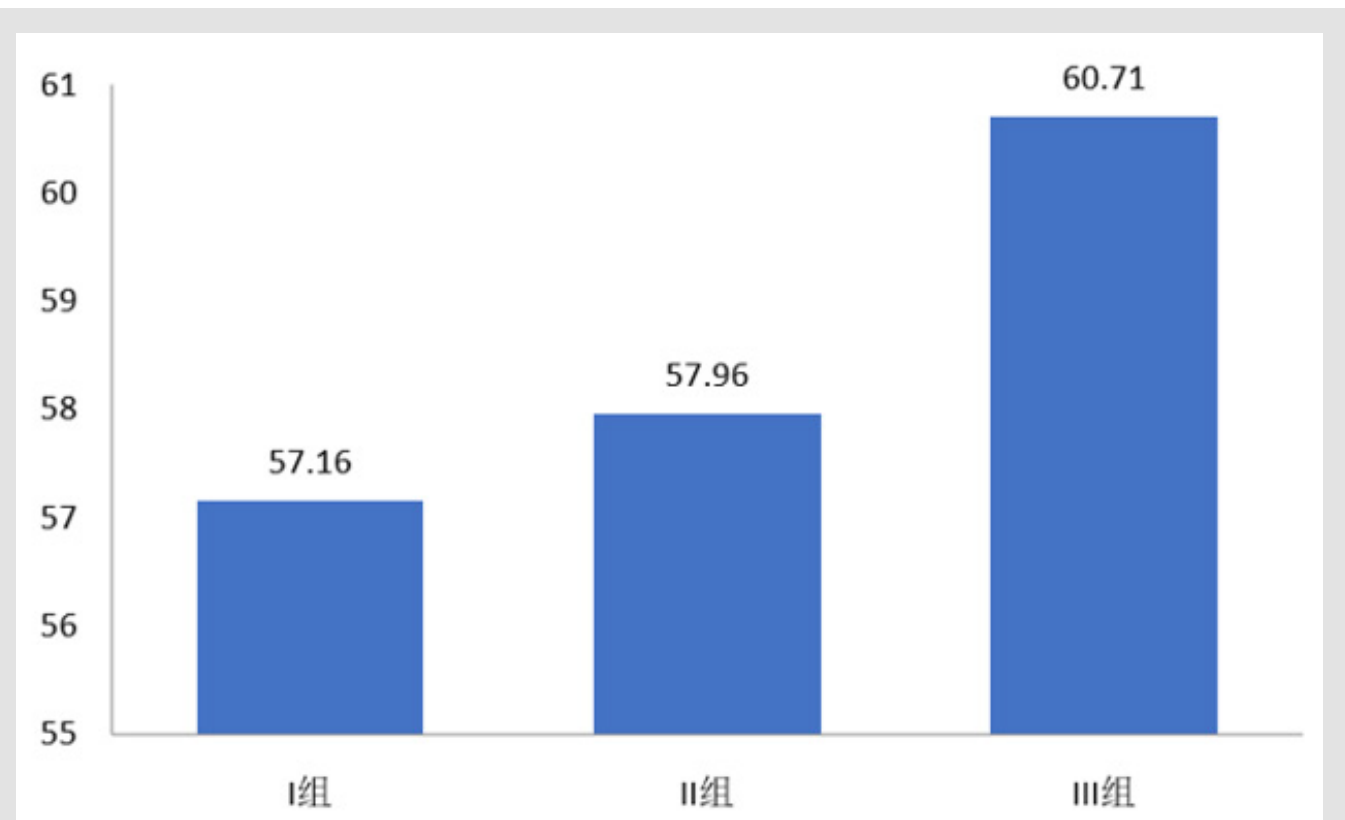

Figure 1: Comparison of coronary Gensini scores among HbA1c 3 groups $(P=0.500)$.

The Relationship between HbA1c and the Degree of Coronary Artery Stenosis

After adjusting for age, gender, hypertension history, smoking history, TC, TG,HDL-C, LDL-C, FBG, Lp(a) and other factors, there was no significant relationship between $\mathrm{HbA1c}$ level and Gensini score (Table 3).

Table 3: Relationship between HbA1c and degree of coronary artery stenosis.

\begin{tabular}{|c|c|c|c|c|}
\hline & Model 1 & Model 2 & Model 3 & Model 4 \\
\hline HbA1cdegreecorrelation & 0.068 & 0.006 & 0.051 & 0.001 \\
\hline P & 0.242 & 0.296 & 0.377 & 0.985 \\
\hline
\end{tabular}

Note: Model 1: Spearman rank correlation analysis;

Model 2: Spearman partial grade correlation analysis, gender and age correction;

Model 3: Spearman partial grade correlation analysis, adjusted for gender, age, hypertension history and smoking history;

Model 4: Spearman partial grade correlation analysis, adjusted for gender, age, history of hypertension, smoking history, TC, TG, hdl-c, ldl-c, FBG, Lp(a).

\section{Relative Analysis of Lp(a) Level and HbA1c Level in Patients}

Spearman correlation analysis was used to analyze Lp (a) level and $\mathrm{HbA} 1 \mathrm{c}$ level. The results showed that there was no correlation between Lp (a) level and HbA1c level ( $r=0.103, \mathrm{P}=0.075)$.

\section{Discussion}

Coronary heart disease (CHD) is a stenosis or occlusion of vascular lumen caused by embolism and inflammation. It is often divided into acute coronary syndrome (ACS) and stable coronary heart disease (SCD). In recent years, due to the change of people's living habits and the aging of the population, the incidence of coronary heart disease has increased year by year. At present, the number of coronary heart disease deaths in China has risen to the second place in the world, seriously threatening Chinese health and even life. However, the etiology is still unclear. Most studies believe that it is caused by multiple genetic and environmental factors, including age, sex, smoking, hyperlipidemia, hypertension, diabetes and impaired glucose tolerance, unhealthy diet, lack of exercise, life stress, genetic factors and so on [7]. Meanwhile, a large number of studies have found that some new factors seem to be related to the occurrence and development of coronary artery diseases, including hemoglobin a1c (HbA1c), lipoprotein a(Lp(a)), bilirubin (TBIL), fibrinogen (Fbg), homocysteine (Hcy), serum uric acid and c-reactive protein (CRP).

Lipoprotein a (Lp(a)) was first discovered and named by the Norwegian scientist Berg et al. [8] in 1963. It is a special lipoprotein synthesized by the liver, mainly composed of LDL and Apo(a), which both through disulfide bond covalency connection. LDL components mainly including cholesterol, phospholipids and ApoB - 100. Apart from genetic factors, they are almost independent of factors such as age, sex, smoking, diet, lipid metabolism, drugs, and environment. Some studies at home and abroad have confirmed that elevated Lp (a) is closely related to coronary heart disease and is considered as a risk factor for coronary heart disease [9]. The pathogenic mechanism mainly has the following several points. 
(1) Lp(a) is highly similar to plasmin (Pg) in structure, and binds fibrin competitively with plasmin, thereby inhibiting the hydrolysis of fibrin and increasing fibrin in plasma. Meanwhile, Lp(a) promotes the formation of thrombosis by inhibiting the activation of t-PA to Pg [10].

(2) Lp (a) contributes to atherosclerosis through regulating the aggregation of single nuclei,activating complement and participating in inflammation.

(3) Lp (a) adheres to vascular endothelial cells, causing endothelial dysfunction, endothelial cell apoptosis, participating in the proliferation of vascular endothelial smooth muscle cells, and leading to the occurrence and development of atherosclerosis.

(4) Lp (a) can pass through the vascular endothelium, deposit in the intima, and combine with the matrix components, thereby resulting in secondary peroxidation.

Chemically modified Lp (a) can enter macrophages, and then transform into foam cells to promote the occurrence and development of AS plaques and thrombosis [11]. A domestic study on the relationship between various factors of blood lipid and atherosclerosis [12] showed that Lp (a) > TG > LDL-C > TC > HDL $>$ apoB $>$ apoA1, suggesting that Lp (a) is superior to other blood lipid indicators in predicting AS and coronary heart disease. This study found that serum Lp (a) level was positively correlated with coronary Gensini score, not affected by age, sex, smoking, blood pressure and other factors, but not significantly correlated with TC, TG, LDL, HDL. Therefore, Lp (a) is an independent risk factor for coronary heart disease, which can be used as a predictor of the severity of coronary artery disease. Glycosylated hemoglobin (HbA1c) is a product of continuous and irreversible non-enzymatic reaction between valine and glucose at the $\mathrm{N}$-terminal of two beta chains in erythrocyte $\mathrm{Hb}$ in blood. It can usually reflect the control of blood sugar for nearly 2-3 months. American Diabetes Association (ADA) and World Health Organization (WHO) have recommended HbA1c content as a new diagnostic criterion for DM, and HbA1c > $6.5 \%$ as a clinical diagnostic cut-off point $[13,14]$. In recent years, a large number of domestic and foreign research data show that $\mathrm{HbA1c}$ is an independent risk factor for coronary heart disease in both diabetic and non-diabetic patients and is positively correlated with the severity of coronary artery disease.

The results of Khaw et al. [15] showed that for every $1 \%$ increase in $\mathrm{HbA1c}$, the psychogenic mortality rates of men and women increased to 1.24 and 1.28, respectively. Saleemt et al. [16] also considered that $\mathrm{HbA} 1 \mathrm{c}$ was a risk factor affecting the degree of coronary artery disease. A meta-analysis showed that [17] glycosylated hemoglobin (HbA1c) was an independent risk factor for mortality in non-diabetic patients with coronary heart disease, but not in patients with diabetes. However, the results of this study are different. The results show that the Gensini score of coronary artery increases with the increase of glycosylated hemoglobin level in non-diabetic patients with coronary heart disease, but there is no statistical significance. But this does not mean lower HbA1c level would bring benefit to patients with cardiovascular. A large study of non-diabetic patients suggested that patients with lower HbA1c levels had better cardiovascular benefits [18]. Therefore, effective control of HbA1c level can still help patients with CHD better benefit. In summary, Lp(a) may be used as a risk factor for coronary heart disease, which is closely related to the degree of coronary artery disease and has a high clinical value in predicting the degree of coronary artery disease. Whether $\mathrm{HbA1c}$ is related to the degree of coronary artery disease needs to be further confirmed by large sample tests.

\section{References}

1. Gerstein HC, Islam S, Anand S, Almahmeed W, Damasceno A, et al. (2015) Dysglycaemia and therisk of acute myocardial infarction in multiple ethnic groups: an analysis of 15780 patients from the INTERHEART study. Diabctologia 53(12): 2509-2517.

2. Teno S, Uto Y, Nagashima H, Endoh Y, Iwamoto Y, et al. (2015) Association of postprandial hy-pertriglyceridemia and carotid INTIMAMEDIATHICKNESS in patientswith type 2 diabetes. Diabetes Care 23(9): 1404-1406.

3. Zhang Wei, Li Mengmeng, Liu Hualing (2018) The relationship between glycosylated hemoglobin, lipoprotein $\mathrm{A}$ and coronary heart disease. Chinese and foreign medical treatment 37(5): 34-36.

4. Zhang Fengxia, Yan Pingping, Zhu Wen, Li Yong, Shi Haiming (2009) Clinical value of glycosylated hemoglobin in patients with non-diabetic coronary heart disease. Chinese Journal of Practical Diagnosis and Treatment 3: 217-219.

5. Tian Lei, Mi Shuhua, Su Gong, Tao Hong, Zhao Quanming, et al. (2007) The relationship between glycosylated hemoglobin level and coronary heart disease in non-diabetic population. Chinese Journal of Diabetes 15(10): 598-600.

6. Cairns BJ, Coffey S, Travis RC, Prendergast B, Green J, et al. (2017) A replicated, genome_wide significant association of aortic stenosis with a genetic variant for lipoprotein (a): meta-analysis of published and novel data. Circulation 135(12): 1181-1183.

7. Yujie Huo Yong, Ge Junbo, et al. (2013) Classic Question and Answer 1000 for Clinical Cardiovascular Diseases. Beijing: People's Health Publishing House p. 1-4.

8. Berg K (1963) A new serum type in man: the LP system. Acta Pathol Microbiol Seand 59: 369-382.

9. Carey VJ, Bishop L, Laranjo N, Harshfield BJ, Kwiat C, et al. (2010) Contribution of high plasma triglycerides and low-high-density lipoprotein cholesterol to residual risk of coronary heart disease after establishment of low-density lipoprotein cholesterol control. Am J Cardiol 106(6): 757-763.

10. Xie Jin, Hu Pei, Li Xin (2017) The relationship between glycosylated hemoglobin, lipoprotein-a and severity of coronary artery disease in patients with coronary atherosclerotic heart disease. Lingnan Journal of Cardiovascular Disease 23(3): 241-244.

11. Lu Yafei (2015) Study on the relationship between glycosylated hemoglobin level and severity of coronary artery disease in patients with coronary heart disease without diabetes. Dalian Medical University.

12. Yang Huijian, Tian Xiangting, Zhong Zhian (2014) The correlation between glycosylated hemoglobin and severity of coronary artery 
disease in patients with coronary heart disease. Modern Hospital 14(6) 31-33.

13. American Diabetes Association (2010) Diagnosis and classification of diabetes mellitus. Diabetes care. 33(1): 62-69.

14. (2011) World Health Organization Use of glycated Haemoglobin (HbA1c) in the diagnosis of diabetes mellitus: abbreviated report of a WHO consultation, Switzerland.

15. Khaw KT, Wareham N, Bingham S, Luben R, Welch A, et al. (2004) Association of haemoglobin A1C with cardiovascular disease and mortality in adulm: The European Prospective Investigation into Cancer in Norfolk. Ann Intern Med 141(6): 413-420.

ISSN: 2574-1241

DOI: 10.26717/BJSTR.2019.21.003624

Qi Chunmei. Biomed J Sci \& Tech Res

This work is licensed under Creative Commons Attribution 4.0 License

Submission Link: https://biomedres.us/submit-manuscript.php
16. Saleem T, Mohammad KH, Abdel Fattah MM, Abbasi AH (2008) Association of glycosylated haemoglobin level and diabetes mellitus duration with the severity of coronary artery disease. Diab Vasc Dis Res 5(3): 184-189.

17. Yao Liu, Yan Min Yang, Jun Zhu, Tan HQ, Liang Y, et al. (2011) Prognostic significance of hemoglobin A1c level in patients hospitalized with coronary artery disease. A systematic review and meta-analysis. Cardiovascular Diabetology 10: 98.

18. Selvin E, Steffes MW, Zhu H, Matsushita K, Wagenknecht L, et al. (2010) Glyeated Hemoglobin, diabetes, and cardiovascular risk in non-diabetic adults. N Engl J Med 362: 800-811.

$\begin{array}{ll}\text { BIOMEDICAL } & \text { Assets of Publishing with us } \\ \text { RESEARCHES } & \text { - Global archiving of articles } \\ & \text { - Immediate, unrestricted online access } \\ & \text { - Rigorous Peer Review Process } \\ & \text { - Authors Retain Copyrights }\end{array}$

